\title{
Fuzzy Stopping Problems in Continuous-Time Fuzzy Stochastic Systems
}

\author{
Y. YOSHIDA ${ }^{\S}$, M. YASUDA ${ }^{\dagger}$, J. NAKAGAMI $^{\dagger}$ and M. KURANO \\ $\S$ Faculty of Economics and Business Administration, the University of Kitakyushu, Koku- \\ raminami, Kitakyushu 802-8577, Japan. ${ }^{\dagger}$ Faculty of Science, Chiba University, ${ }^{\ddagger}$ Faculty of \\ Education, Yayoi-cho,Inage-ku,Chiba 263-8522, Japan.
}

\begin{abstract}
In a continuous-time fuzzy stochastic system, a stopping model with fuzzy stopping times is presented. The optimal fuzzy stopping times are given under an assumption of regularity for stopping rules. Also, the optimal fuzzy reward is characterized as a unique solution of an optimality equation under a differentiability condition. An example in the Markov models is discussed.
\end{abstract}

Keywords: Fuzzy stopping; Continuous-time stopping model; Fuzzy stochastic system; Optimal stopping time; Optimality equation.

\section{Introduction}

Stopping problems for a sequence of 'real-valued' random variables were studied by many authors, and their applications are well known in various fields, management science, finance, engineering etc.(Presman and Sonin [6], Shiryayev [9]). This paper discusses 'optimal fuzzy stopping in a continuous-time fuzzy stochastic system' defined by fuzzy random variables.

The fuzzy random variable, which is a 'fuzzy-number-valued' extension of real random variables, was first studied by Puri and Ralescu [7] and has been discussed by many authors. By introducing fuzziness to stochastic processes in optimization/decision-making, we consider an optimal stopping model with uncertainty of both randomness and fuzziness, which is a reasonable and natural extension of the original stochastic processes. In stochastic systems, the randomness is a notion of uncertainty which implies whether something occurs or not with some probability, and, in this paper, the fuzziness means an uncertainty like ambiguity where we can not identify exact values because of a lack of knowledge in systems. We, here, deal with them as different kinds of uncertainty.

Fuzzy stopping times are introduced by Kurano et al. [5] in discrete-time dynamic fuzzy systems([4]). [5] has investigated discrete-time One-Step Look Ahead Property of the fuzzy stopping times since the dynamic fuzzy systems are non-stochastic Markov fuzzy systems. This paper extends the definition of fuzzy stopping times to continuous-time and stochastic environments and we discuss them in general framework of continuous-time fuzzy stochastic systems. Recently, in fuzzy stochastic systems defined by sequences of 
fuzzy random variables, Yoshida et al. [13] has studied discrete-time stopping problems in comparison between 'fuzzy stopping times' and non-fuzzy stopping times and has shown that the fuzzy stopping model is more favorite than the non-fuzzy one. This paper also extends the definition of fuzzy stopping times in the discrete-time models $([13])$ to the continuous-time case, and presents a fuzzy stopping model in a continuous-time 'fuzzy stochastic systems' which is constructed from fuzzy random variables. In Section 2, the notations and definitions of fuzzy random variables are given and a continuous-time fuzzy stochastic system is formulated. Next, in Section 3, fuzzy stopping times are introduced for continuous-time fuzzy stochastic systems, and a stopping model by fuzzy stopping times is presented. In Section 4, in the associated stopping model for fuzzy stochastic systems, an optimal fuzzy stopping time is constructed under a regularity assumption regarding stopping rules. In Section 5, it is shown that the optimal fuzzy reward is a unique solution of an optimality equation under a differentiability condition. Finally, in the last section, an example where an owner of an asset finds an optimal timing to sell his own asset, is given to illustrate our idea in the Markov models.

\section{Fuzzy stochastic systems}

First, we introduce some notations of fuzzy numbers and fuzzy random variables. Let $(\Omega, \mathcal{M}, P)$ be a probability space, where $\mathcal{M}$ is a $\sigma$-field and $P$ is a non-atomic probability measure. Let $\mathbb{R}$ be the set of all real numbers. A 'fuzzy number' is denoted by its membership function $\tilde{a}: \mathbb{R} \mapsto[0,1]$ which is normal, upper-semicontinuous, fuzzy convex and has a compact support. Refer to Zadeh [14] for the theory of fuzzy sets. $\mathcal{R}$ denotes the set of all fuzzy numbers. The $\alpha$-cut of a fuzzy number $\tilde{a}(\in \mathcal{R})$ is given by

$$
\tilde{a}_{\alpha}:=\{x \in \mathbb{R} \mid \tilde{a}(x) \geq \alpha\}(\alpha \in(0,1]) \quad \text { and } \quad \tilde{a}_{0}:=\operatorname{cl}\{x \in \mathbb{R} \mid \tilde{a}(x)>0\},
$$

where $\mathrm{cl}$ denotes the closure of an interval. In this paper, we write the closed intervals by

$$
\tilde{a}_{\alpha}:=\left[\tilde{a}_{\alpha}^{-}, \tilde{a}_{\alpha}^{+}\right] \text {for } \alpha \in[0,1] .
$$

We use a metric $\delta_{\infty}$ on $\mathcal{R}$ defined by

$$
\delta_{\infty}(\tilde{a}, \tilde{b}):=\sup _{\alpha \in[0,1]} \delta\left(\tilde{a}_{\alpha}, \tilde{b}_{\alpha}\right) \quad \text { for } \tilde{a}, \tilde{b} \in \mathcal{R},
$$

where $\delta$ is the Hausdorff metric on $\mathbb{R}$. A fuzzy-number-valued map $\tilde{X}: \Omega \mapsto \mathcal{R}$ is called a 'fuzzy random variable' if

$$
\{(\omega, x) \in \Omega \times \mathbb{R} \mid \tilde{X}(\omega)(x) \geq \alpha\} \in \mathcal{M} \times \mathcal{B} \text { for all } \alpha \in[0,1],
$$

where $\mathcal{B}$ is the Borel $\sigma$-field of $\mathbb{R}$. We can find some equivalent conditions in general cases $([7])$, however this paper adopts a simple characterization in the following lemma.

Lemma 2.1 (Wang and Zhang [11, Theorems 2.1 and 2.2]). For a map $\tilde{X}: \Omega \mapsto \mathcal{R}$, the following (i) and (ii) are equivalent: 
(i) $\tilde{X}$ is a fuzzy random variable.

(ii) The maps $\omega \mapsto \tilde{X}_{\alpha}^{-}(\omega)$ and $\omega \mapsto \tilde{X}_{\alpha}^{+}(\omega)$ are measurable for all $\alpha \in[0,1]$, where $\tilde{X}_{\alpha}(\omega)=\left[\tilde{X}_{\alpha}^{-}(\omega), \tilde{X}_{\alpha}^{+}(\omega)\right]:=\{x \in \mathbb{R} \mid \tilde{X}(\omega)(x) \geq \alpha\}$.

Now we introduce expectations of fuzzy random variables to describe stopping models in fuzzy stochastic systems. A fuzzy random variable $\tilde{X}$ is called integrably bounded if $\omega \mapsto \tilde{X}_{\alpha}^{-}(\omega)$ and $\omega \mapsto \tilde{X}_{\alpha}^{+}(\omega)$ are integrable for all $\alpha \in[0,1]$. For an integrably bounded fuzzy random variables $\tilde{X}$, we put closed intervals

$$
E(\tilde{X})_{\alpha}:=\left[\int_{\Omega} \tilde{X}_{\alpha}^{-}(\omega) \mathrm{d} P(\omega), \int_{\Omega} \tilde{X}_{\alpha}^{+}(\omega) \mathrm{d} P(\omega)\right], \quad \alpha \in[0,1] .
$$

Then, the expectation $E(\tilde{X})$ of the fuzzy random variable $\tilde{X}$ is defined by a fuzzy number ([4, Lemma 3],[13]):

$$
E(\tilde{X})(x):=\sup _{\alpha \in[0,1]} \min \left\{\alpha, 1_{E(\tilde{X})_{\alpha}}(x)\right\} \quad \text { for } x \in \mathbb{R},
$$

where $1_{D}$ is the indicator function of a set $D$.

Next, we formulate fuzzy stochastic systems. Let $[0, \infty)$ be the time space, and let $\left\{\tilde{X}_{t}\right\}_{t \geq 0}$ be a process of integrably bounded fuzzy random variables such that $E\left(\sup _{t \geq 0} \tilde{X}_{t, 0}^{+}\right)$ $<\infty$, where $\tilde{X}_{t, 0}^{+}(\omega)$ is the right-end of the 0 -cut of the fuzzy number $\tilde{X}_{t}(\omega)$ for $t \geq 0$. We assume that the map $t \mapsto \tilde{X}_{t}(\omega)(\in \mathcal{R})$ is continuous on $[0, \infty)$ for almost all $\omega \in \Omega$. $\left\{\mathcal{M}_{t}\right\}_{t \geq 0}$ is a family of nondecreasing sub- $\sigma$-fields of $\mathcal{M}$ which is right continuous, i.e. $\mathcal{M}_{t}=\bigcap_{r: r>t} \mathcal{M}_{r}$ for all $t \geq 0$, and fuzzy random variables $\tilde{X}_{t}$ are $\mathcal{M}_{t}$-adapted, i.e. random variables $\tilde{X}_{r, \alpha}^{-}$and $\tilde{X}_{r, \alpha}^{+}(0 \leq r \leq t ; \alpha \in[0,1])$ are $\mathcal{M}_{t}$-measurable. And $\mathcal{M}_{\infty}$ denotes the smallest $\sigma$-field containing $\bigcup_{t \geq 0} \mathcal{M}_{t}$. Then $\left(\tilde{X}_{t}, \mathcal{M}_{t}\right)_{t \geq 0}$ is called a continuous-time 'fuzzy stochastic system'. A map $\tau: \Omega \mapsto[0, \infty]$ is said to be a 'stopping time' if

$$
\{\omega \in \Omega \mid \tau(\omega) \leq t\} \in \mathcal{M}_{t} \text { for all } t \geq 0 .
$$

Then we have the following lemma.

Lemma 2.2. Let $\tau$ be a finite stopping time. Define

$$
\tilde{X}_{\tau}(\omega):=\tilde{X}_{\tau(\omega)}(\omega) \text { for } \omega \in \Omega .
$$

Then, $\tilde{X}_{\tau}$ is a fuzzy random variable.

Proof. Let $\alpha \in[0,1]$. The maps $\omega \mapsto \tilde{X}_{t, \alpha}^{ \pm}(\omega)$ are measurable for $t \geq 0$, and the maps $t \mapsto \tilde{X}_{t, \alpha}^{ \pm}(\cdot)$ are right continuous and have left-hand limits almost surely. Since $\tau$ is a stopping time, the maps $\omega \mapsto \tilde{X}_{\tau(\omega), \alpha}^{ \pm}(\omega)$ are measurable. Therefore the proof is completed from Lemma 2.1 .

Here, $\tilde{X}_{\tau}(\omega), \omega \in \Omega$, means a 'fuzzy reward' at a stopping time $\tau$, and the fuzziness indicates ill-conditions where we have a lack of knowledge about them(Kurano et al. [5]). 
The optimal stopping problems for fuzzy rewards in a discrete-time case have been discussed by Yoshida et al. [13]. This paper discusses an optimal stopping problem regarding fuzzy rewards, using fuzzy stopping times, in the next section.

\section{A fuzzy stopping model}

In this section, first we consider an evaluation of the fuzzy stochastic system $\left(\tilde{X}_{t}, \mathcal{M}_{t}\right)_{t \geq 0}$ which is defined in Section 2. at a classical finite stopping time $\tau$ defined by (2.5). Next we introduce a 'fuzzy stopping time' in accordance with the continuous-time fuzzy stochastic system, and we discuss a stopping problem by using fuzzy stopping times. Let $\mathcal{I}$ be the set of all bounded closed sub-intervals of $\mathbb{R}$ and let $g: \mathcal{I} \mapsto \mathbb{R}$ be a continuous $\sigma$-additively homogeneous map, that is, $g$ satisfies the following (3.1) and (3.2):

$$
g\left(\sum_{n=0}^{\infty} c_{n}\right)=\sum_{n=0}^{\infty} g\left(c_{n}\right)
$$

for bounded closed intervals $\left\{c_{n}\right\}_{n=0}^{\infty} \subset \mathcal{I}$ such that $\sum_{n=0}^{\infty} c_{n} \in \mathcal{I}$ and

$$
g(\mu c)=\mu g(c)
$$

for bounded closed intervals $c \in \mathcal{I}$ and real numbers $\mu \geq 0$, where the operation on closed intervals is defined ordinary as $\sum_{n=0}^{\infty} c_{n}:=\operatorname{cl}\left\{\sum_{n=0}^{\infty} x_{n} \mid x_{n} \in c_{n}, n=0,1,2, \cdots\right\}$ and $\mu c:=\{\mu x \mid x \in c\}$. We call this scalarization satisfying (3.1) and (3.2) a 'linear ranking function', and it is used for the evaluation of fuzzy numbers (Fortemps and Roubens [8]). For example, $g([a, b])=\lambda a+(1-\lambda) b$ for $[a, b] \in \mathcal{I}$, where $\lambda \in[0,1]$, is a linear ranking function, and then $\lambda$ is called a 'pessimistic-optimistic index'([10]). We introduce an evaluation of the fuzzy random variable $\tilde{X}_{\tau}$ provided that $\tau$ is a finite stopping time as follows: Let $\omega \in \Omega$. From (2.6), the $\alpha$-cut of the fuzzy number $\tilde{X}_{\tau}(\omega)$ is a closed interval $\tilde{X}_{\tau(\omega), \alpha}(\omega)$, and the expectation is given by the closed interval $E\left(\tilde{X}_{\tau, \alpha}\right)$ from the definition (2.3). Using the linear ranking function $g$, we estimate it by $g\left(E\left(\tilde{X}_{\tau, \alpha}\right)\right)$. Therefore, the evaluation of the fuzzy random variable $\tilde{X}_{\tau}$ is given by the integral

$$
G_{\tau}(\omega):=\int_{0}^{1} g\left(E\left(\tilde{X}_{\tau, \alpha}\right)\right) \mathrm{d} \alpha .
$$

This means an 'evaluation of the fuzzy reward at a stopping time $\tau$ '. Then we have the following lemma regarding (3.3).

Lemma 3.1. For a finite stopping time $\tau$, it holds that

$$
\int_{0}^{1} g\left(E\left(\tilde{X}_{\tau, \alpha}\right)\right) d \alpha=\int_{0}^{1} E\left(g\left(\tilde{X}_{\tau, \alpha}\right)\right) d \alpha=E\left(\int_{0}^{1} g\left(\tilde{X}_{\tau, \alpha}(\cdot)\right) d \alpha\right)=E\left(G_{\tau}\right) .
$$


Proof. The properties (3.1) and (3.2) of $g$ imply $g\left(E\left(\tilde{X}_{\tau, \alpha}\right)\right)=E\left(g\left(\tilde{X}_{\tau, \alpha}\right)\right)$. Therefore, by Fubini's theorem, we obtain (3.4).

Next we introduce fuzzy stopping times, which is a fuzzification of classical stopping times (2.5) and is also a continuous-time extension of fuzzy stopping times in [13].

Definition 3.1. A map $\tilde{\tau}:[0, \infty) \times \Omega \mapsto[0,1]$ is called a fuzzy stopping time if it satisfies the following (i) - (iii):

(i) For each $t \geq 0$, the map $\omega \mapsto \tilde{\tau}(t, \omega)$ is $\mathcal{M}_{t}$-measurable.

(ii) For almost all $\omega \in \Omega$, the map $t \mapsto \tilde{\tau}(t, \omega)$ is non-increasing and right continuous and has left-hand limits on $[0, \infty)$.

(iii) For almost all $\omega \in \Omega$, there exists $t_{0} \geq 0$ such that $\tilde{\tau}(t, \omega)=0$ for all $t \geq t_{0}$.

Definition 3.1 is the similar idea to fuzzy stopping times given in dynamic fuzzy systems by Kurano et al. [5]. Regarding the membership grade of fuzzy stopping times, $\tilde{\tau}(t, \omega)=0$ means 'to stop at time $t$ ' and $\tilde{\tau}(t, \omega)=1$ means 'to continue at time $t$ ' respectively. We have the following lemma regarding the properties of fuzzy stopping times.

\section{Lemma 3.2.}

(i) Let $\tilde{\tau}$ be a fuzzy stopping time. Define a map $\tilde{\tau}_{\alpha}: \Omega \mapsto[0, \infty)$ by

$$
\tilde{\tau}_{\alpha}(\omega):=\inf \{t \geq 0 \mid \tilde{\tau}(t, \omega)<\alpha\}, \quad \omega \in \Omega \quad \text { for } \alpha \in(0,1]
$$

where the infimum of the empty set is understood to be $+\infty$. Then, we have:

(a) $\left\{\omega \mid \tilde{\tau}_{\alpha}(\omega) \leq t\right\} \in \mathcal{M}_{t}$ for $t \geq 0$;

(b) $\tilde{\tau}_{\alpha}(\omega) \leq \tilde{\tau}_{\alpha^{\prime}}(\omega)$ for almost all $\omega \in \Omega$ if $\alpha \geq \alpha^{\prime}$;

(c) $\lim _{\alpha^{\prime} \uparrow \alpha} \tilde{\tau}_{\alpha^{\prime}}(\omega)=\tilde{\tau}_{\alpha}(\omega)$ for almost all $\omega \in \Omega$ if $\alpha>0$;

(d) $\tilde{\tau}_{0}(\omega):=\lim _{\alpha \downarrow 0} \tilde{\tau}_{\alpha}(\omega)<\infty$ for almost all $\omega \in \Omega$.

(ii) Let $\left\{\tilde{\tau}_{\alpha}\right\}_{\alpha \in[0,1]}$ be maps $\tilde{\tau}_{\alpha}: \Omega \mapsto[0, \infty)$ satisfying the above (a) (b) and (d). Define a map $\tilde{\tau}:[0, \infty) \times \Omega \mapsto[0,1]$ by

$$
\tilde{\tau}(t, \omega):=\sup _{\alpha \in[0,1]} \min \left\{\alpha, 1_{\left\{\tilde{\tau}_{\alpha}>t\right\}}(\omega)\right\} \quad \text { for } t \geq 0 \text { and } \omega \in \Omega .
$$

Then $\tilde{\tau}$ is a fuzzy stopping time.

Proof. For (i), (a),(b) and (d) are trivial from Definition 3.1. From (b), we have

$$
\lim _{\alpha^{\prime} \uparrow \alpha} \tilde{\tau}_{\alpha^{\prime}}(\omega)=\inf _{\alpha^{\prime}: \alpha^{\prime}<\alpha} \inf \left\{t \geq 0 \mid \tilde{\tau}(t, \omega)<\alpha^{\prime}\right\}=\inf \{t \geq 0 \mid \tilde{\tau}(t, \omega)<\alpha\}=\tilde{\tau}_{\alpha}(\omega),
$$


which implies (c). For (ii), it is sufficient to check Definition 3.1(ii) about (3.6) since (3.6) trivially satisfies the other conditions of Definition 3.1. The map $t \mapsto \tilde{\tau}(t, \omega)$ is clearly non-increasing. Further we have

$$
\begin{aligned}
\lim _{t^{\prime} \downarrow t} \tilde{\tau}\left(t^{\prime}, \omega\right) & =\lim _{t^{\prime} \downarrow t} \sup _{\alpha \in[0,1]} \min \left\{\alpha, 1_{\left\{\tilde{\tau}_{\alpha}>t^{\prime}\right\}}(\omega)\right\} \\
& =\sup _{t^{\prime}: t^{\prime}>t} \sup _{\alpha \in[0,1]} \min \left\{\alpha, 1_{\left\{\tilde{\tau}_{\alpha}>t^{\prime}\right\}}(\omega)\right\} \\
& =\sup _{\alpha \in[0,1]} \sup _{t^{\prime}: t^{\prime}>t} \min \left\{\alpha, 1_{\left\{\tilde{\tau}_{\alpha}>t^{\prime}\right\}}(\omega)\right\} \\
& =\sup _{\alpha \in[0,1]} \min \left\{\alpha, 1_{\left\{\tilde{\tau}_{\alpha}>t\right\}}(\omega)\right\} \\
& =\tilde{\tau}(t, \omega) .
\end{aligned}
$$

Therefore the map $t \mapsto \tilde{\tau}(t, \omega)$ is right continuous and has left-hand limits from the monotonicity. These complete the proof of this lemma.

Now we consider the estimation of the fuzzy stochastic system stopped at a 'fuzzy stopping time $\tilde{\tau}^{\prime}$. Since it is difficult to define the fuzzy random variable stopped at fuzzy stopping times $\tilde{\tau}$ in the way of (2.6), we establish it by $\alpha$-cut technique of fuzzy random variables and fuzzy stopping times. A fuzzy stopping time $\tilde{\tau}$ is called finite if $\tilde{\tau}_{0}(\omega):=\lim _{\alpha \downarrow 0} \tilde{\tau}_{\alpha}(\omega)<\infty$ for almost all $\omega \in \Omega$. Let $\tilde{\tau}$ be a finite fuzzy stopping time. Then, from Lemma 3.2(i.a), $\tilde{\tau}_{\alpha}$ is a 'classical bounded stopping time' given by (3.5). Let $\omega \in \Omega . \quad \tilde{X}_{\tilde{\tau}_{\alpha}, \alpha}(\omega):=\tilde{X}_{\tilde{\tau}_{\alpha}(\omega), \alpha}(\omega)$ corresponds to the $\alpha$-cut of the fuzzy stochastic system $\left\{\tilde{X}_{t}\right\}_{t \geq 0}$ stopped at fuzzy stopping time $\tilde{\tau}$. Therefore, by the evaluation method in (3.3), we define a random variable

$$
G_{\tilde{\tau}}(\omega):=\int_{0}^{1} g\left(\tilde{X}_{\tilde{\tau}_{\alpha}, \alpha}(\omega)\right) \mathrm{d} \alpha, \quad \omega \in \Omega .
$$

The expectation $E\left(G_{\tilde{\tau}}\right)$ is the evaluation of the fuzzy random variable $\tilde{X}_{\tilde{\tau}}$, and it means an 'evaluation of the fuzzy reward at a fuzzy stopping time $\tilde{\tau}$ '. We note that if $\tilde{\tau}$ is corresponding to a non-fuzzy (classical) stopping time $\tau$, that is,

$$
\tilde{\tau}(t, \omega)= \begin{cases}1 & \text { if } t<\tau(\omega) \\ 0 & \text { if } t \geq \tau(\omega)\end{cases}
$$

it holds that $E\left(G_{\tilde{\tau}}\right)=E\left(G_{\tau}\right)$. In this paper, we discuss the following problem.

Problem 1. Find a fuzzy stopping time $\tilde{\tau}^{*}$ such that $E\left(G_{\tilde{\tau}^{*}}\right) \geq E\left(G_{\tilde{\tau}}\right)$ for all fuzzy stopping times $\tilde{\tau}$.

In Problem 1, $\tilde{\tau}^{*}$ is called an 'optimal fuzzy stopping time'. Now, by Lemma 3.1, we have

$$
E\left(G_{\tilde{\tau}}\right):=E\left(\int_{0}^{1} g\left(\tilde{X}_{\tilde{\tau}_{\alpha}, \alpha}(\cdot)\right) \mathrm{d} \alpha\right)=\int_{0}^{1} E\left(g\left(\tilde{X}_{\tilde{\tau}_{\alpha}, \alpha}\right)\right) \mathrm{d} \alpha
$$


for fuzzy stopping times $\tilde{\tau}$. In order to analyze Problem 1, we need to discuss the following subproblem induced from (3.8).

Problem 2. Let $\alpha \in[0,1]$. Find a stopping time $\tau^{*}$ such that $E\left(g\left(\tilde{X}_{\tau^{*}, \alpha}\right)\right) \geq E\left(g\left(\tilde{X}_{\tau, \alpha}\right)\right)$ for all stopping times $\tau$.

In Problem $2, \tau^{*}$ is called an ' $\alpha$-optimal stopping time'.

\section{An optimal fuzzy stopping time}

This section is devoted to a method to construct an optimal fuzzy stopping time. In order to characterize $\alpha$-optimal stopping times, we let

$$
U_{t}^{\alpha}:=\underset{\tau: \text { stopping times, } \tau \geq t}{\operatorname{ess} \sup } E\left(g\left(\tilde{X}_{\tau, \alpha}\right) \mid \mathcal{M}_{t}\right) \quad \text { for } t \geq 0,
$$

where 'ess sup' means the essential supremum([9]). Then $U_{t}^{\alpha}$ are right continuous with respect to $t \geq 0$ since $\tilde{X}_{t, \alpha}$ and $\mathcal{M}_{t}$ are right continuous with respect to $t \geq 0$ and $g$ is continuous. We define a stopping time $\sigma_{\alpha}^{*}: \Omega \mapsto[0, \infty)$ by

$$
\sigma_{\alpha}^{*}(\omega):=\inf \left\{t \geq 0 \mid U_{t}^{\alpha}(\omega)=g\left(\tilde{X}_{t, \alpha}(\omega)\right)\right\}
$$

for $\omega \in \Omega$ and $\alpha \in[0,1]$, where the infimum of the empty set is understood to be $+\infty$. The stopping time (4.2) is a general form of the first hitting time of the optimal stopping region(See Example 6.1). Then, Problem 2 is one of classical stopping problems in continuous-time stochastic processes and we can find the proof of the next Theorem 4.1 in [2] and [9, Theorem 3 in Sect.3.3.3], but we omit the proof because it is long.

Theorem 4.1. Let $\alpha \in[0,1]$. If $\sigma_{\alpha}^{*}$ is finite almost surely, then $\sigma_{\alpha}^{*}$ is $\alpha$-optimal and $E\left(U_{0}^{\alpha}\right)=E\left(g\left(\tilde{X}_{\sigma_{\alpha}^{*}, \alpha}\right)\right)$.

In order to construct an optimal fuzzy stopping time from the $\alpha$-optimal stopping times $\left\{\sigma_{\alpha}^{*}\right\}_{\alpha \in[0,1]}$, we need the following regularity condition.

Assumption A (Regularity). The map $\alpha \mapsto \sigma_{\alpha}^{*}(\omega)$ is non-increasing for almost all $\omega \in \Omega$.

It depends on the form of the linear ranking function $g$ in actual cases whether Assumption A is satisfied or violated (See Section 6). Under Assumption A, we can define a map $\tilde{\sigma}^{*}:[0, \infty) \times \Omega \mapsto[0,1]$ by

$$
\tilde{\sigma}^{*}(t, \omega):=\sup _{\alpha \in[0,1]} \min \left\{\alpha, 1_{\left\{\sigma_{\alpha}^{*}>t\right\}}(\omega)\right\} \quad \text { for } t \geq 0 \text { and } \omega \in \Omega .
$$

Let $\omega \in \Omega$. For a fuzzy stopping time $\tilde{\sigma}^{*}(t, \omega)$, we denote its $\alpha$-cut in the form (3.5) by $\tilde{\sigma}_{\alpha}^{*}(\omega)$. Then we note that $\tilde{\sigma}_{\alpha}^{*}(\omega)$ and $\sigma_{\alpha}^{*}(\omega)$ are equal except at most countable many $\alpha \in(0,1]$. 
Theorem 4.2 (Optimal fuzzy stopping time). Suppose Assumption A holds. If $P\left(\tilde{\sigma}_{0}^{*}<\right.$ $\infty)=1$, then $\tilde{\sigma}^{*}$ is an optimal fuzzy stopping time for Problem 1. Further it holds that

$$
\tilde{\sigma}_{\alpha}^{*}(\omega)=\min \left\{t \geq 0 \mid \tilde{\sigma}^{*}(t, \omega)<\alpha\right\}, \quad \omega \in \Omega \quad \text { for } \alpha \in(0,1] .
$$

Proof. From Assumption A and Lemma 3.2, $\tilde{\sigma}^{*}$ is a fuzzy stopping time. By (3.8), (4.1) and Theorem 4.1, we have

$$
E\left(G_{\tilde{\tau}}\right) \leq \int_{0}^{1} \sup _{\tau: \text { stopping times }} E\left(g\left(\tilde{X}_{\tau, \alpha}\right)\right) \mathrm{d} \alpha=\int_{0}^{1} E\left(U_{0}^{\alpha}\right) \mathrm{d} \alpha=\int_{0}^{1} E\left(g\left(\tilde{X}_{\sigma_{\alpha}^{*}, \alpha}\right)\right) \mathrm{d} \alpha
$$

for all fuzzy stopping times $\tilde{\tau}$. Since $\tilde{\sigma}_{\alpha}^{*}(\omega) \neq \sigma_{\alpha}^{*}(\omega)$ holds only at most countable $\alpha \in$ $(0,1]$

$$
\int_{0}^{1} g\left(\tilde{X}_{\sigma_{\alpha}^{*}, \alpha}(\omega)\right) \mathrm{d} \alpha=\int_{0}^{1} g\left(\tilde{X}_{\tilde{\sigma}_{\alpha}^{*}, \alpha}(\omega)\right) \mathrm{d} \alpha
$$

holds for almost all $\omega \in \Omega$. By Fubini's theorem, we get

$$
\int_{0}^{1} E\left(g\left(\tilde{X}_{\sigma_{\alpha}^{*}, \alpha}\right)\right) \mathrm{d} \alpha=\int_{0}^{1} E\left(g\left(\tilde{X}_{\tilde{\sigma}_{\alpha}^{*}, \alpha}\right)\right) \mathrm{d} \alpha .
$$

By (4.5) and (4.6), we obtain

$$
E\left(G_{\tilde{\tau}}\right) \leq \int_{0}^{1} E\left(g\left(\tilde{X}_{\sigma_{\alpha}^{*}, \alpha}\right)\right) \mathrm{d} \alpha=\int_{0}^{1} E\left(g\left(\tilde{X}_{\tilde{\sigma}_{\alpha}^{*}, \alpha}\right)\right) \mathrm{d} \alpha=E\left(G_{\tilde{\sigma}^{*}}\right) .
$$

Therefore $\tilde{\sigma}^{*}$ is optimal for Problem 1. Finally, (4.4) holds trivially from Lemma 3.2.

The following result implies a comparison between the optimal values of the 'classical' stopping model and the 'fuzzy' stopping model (Problem 1). Then we find that the fuzzy stopping model is more better than the classical one. This fact has been explicitly shown in the discrete-time model by [13].

Corollary 4.1. It holds that, under the same assumptions as Theorem 4.2,

$$
E\left(G_{\tau^{*}}\right) \leq E\left(G_{\tilde{\sigma}^{*}}\right)
$$

where $\tilde{\sigma}^{*}$ is the optimal fuzzy stopping time and $\tau^{*}$ is an optimal stopping time in the class of classical stopping times.

Proof. For all stopping times $\tau$, from (4.5) and (4.7) we have

$$
E\left(G_{\tau}\right)=E\left(\int_{0}^{1} g\left(\tilde{X}_{\tau, \alpha}\right) \mathrm{d} \alpha\right) \leq \int_{0}^{1} \sup _{\tau} E\left(g\left(\tilde{X}_{\tau, \alpha}\right)\right) \mathrm{d} \alpha=E\left(G_{\tilde{\sigma}^{*}}\right) .
$$

Therefore this corollary holds. 


\section{Optimality equations}

In this section, we consider the optimality conditions for the optimal rewards $\left\{U_{t}^{\alpha}\right\}_{t \geq 0}$. The optimality characterization of optimal rewards has been studied by Shiryayev [9] in stochastic processes, and it has also been discussed by Yoshida [12] in fuzzy deterministic systems. Now, in fuzzy stochastic systems, we derive optimality conditions and optimality equations with a differential operator by a similar idea on the basis of dynamic programming approach.

Theorem 5.1 (Optimality characterization). For $\alpha \in[0,1]$ and $t \geq 0$, the following (i) - (iii) hold:

(i) For almost all $\omega \in \Omega$, it holds that

$$
U_{t}^{\alpha}(\omega) \geq g\left(\tilde{X}_{t, \alpha}(\omega)\right)
$$

(ii) For almost all $\omega \in \Omega$, it holds that

$$
U_{t}^{\alpha}(\omega) \geq E\left(U_{r}^{\alpha} \mid \mathcal{M}_{t}\right)(\omega), \quad r \in[t, \infty) .
$$

(iii) For almost all $\omega \in \Omega$ satisfying $U_{t}^{\alpha}(\omega)>g\left(\tilde{X}_{t, \alpha}(\omega)\right)$, there exists $\varepsilon>0$ such that

$$
U_{t}^{\alpha}(\omega)=E\left(U_{r}^{\alpha} \mid \mathcal{M}_{t}\right)(\omega), \quad r \in[t, t+\varepsilon) .
$$

Proof. (i) We have $U_{t}^{\alpha}=\operatorname{ess}_{\sup _{\tau: \tau \geq t}} E\left(g\left(\tilde{X}_{\tau, \alpha}\right) \mid \mathcal{M}_{t}\right)$ from the definition (4.1). Then particularly by considering the case of $\tau=t$, it holds that $U_{t}^{\alpha} \geq E\left(g\left(\tilde{X}_{t, \alpha}\right) \mid \mathcal{M}_{t}\right)=g\left(\tilde{X}_{t, \alpha}\right)$ almost surely since $g\left(\tilde{X}_{t, \alpha}\right)$ is $\mathcal{M}_{t}$-measurable. (ii) Let $t, r \in[0, \infty)$ satisfy $t \leq r$. From the definition of fuzzy conditional expectation and the monotone convergence theorem, we have

$$
\begin{aligned}
E\left(U_{r}^{\alpha} \mid \mathcal{M}_{t}\right) & =E\left(\operatorname{ess}_{\tau: \tau \geq r} E\left(g\left(\tilde{X}_{\tau, \alpha}\right) \mid \mathcal{M}_{r}\right) \mid \mathcal{M}_{t}\right) \\
& =\operatorname{ess}_{\tau: \tau \geq r} E\left(E\left(g\left(\tilde{X}_{\tau, \alpha}\right) \mid \mathcal{M}_{r}\right) \mid \mathcal{M}_{t}\right) \\
& =\operatorname{ess}_{\tau: \tau \geq r} E\left(g\left(\tilde{X}_{\tau, \alpha}\right) \mid \mathcal{M}_{t}\right) \\
& \leq \underset{\tau \sup }{\operatorname{ess}} E\left(g\left(\tilde{X}_{\tau, \alpha}\right) \mid \mathcal{M}_{t}\right) \\
& =U_{t}^{\alpha} \quad \text { almost surely. }
\end{aligned}
$$

(iii) If $U_{t}^{\alpha}(\omega)>g\left(\tilde{X}_{t, \alpha}(\omega)\right)$ for some $\omega$, then there exists a real number $\varepsilon>0$ and a real random variable $\eta(\omega)>0$ such that $U_{r^{\prime}}^{\alpha}(\omega)>g\left(\tilde{X}_{r^{\prime}, \alpha}(\omega)\right)+\eta(\omega)$ for all $r^{\prime} \in[t, t+\varepsilon)$ since the processes are right continuous. So, by (i) and (ii) we obtain

$$
U_{t}^{\alpha}(\omega) \geq E\left(U_{r^{\prime}}^{\alpha} \mid \mathcal{M}_{t}\right)(\omega)>E\left(g\left(\tilde{X}_{r^{\prime}, \alpha}\right) \mid \mathcal{M}_{t}\right)(\omega)+\eta^{\prime}(\omega) \text { for all } r^{\prime} \in[t, t+\varepsilon),
$$


where $\eta^{\prime}(\omega):=E\left(\eta \cdot 1_{\Gamma} \mid \mathcal{M}_{t}\right)(\omega)>0$ and $\Gamma:=\left\{\omega^{\prime} \mid U_{r^{\prime}}^{\alpha}\left(\omega^{\prime}\right)>g\left(\tilde{X}_{r^{\prime}, \alpha}\left(\omega^{\prime}\right)\right)+\eta\left(\omega^{\prime}\right)\right\}$. It follows

$$
U_{t}^{\alpha}(\omega) \geq \operatorname{ess}_{\tau: t \leq \tau<t+\varepsilon} E\left(g\left(\tilde{X}_{\tau, \alpha} \mid \mathcal{M}_{t}\right)(\omega)+\eta^{\prime}(\omega)>\operatorname{ess} \sup _{\tau: t \leq \tau<t+\varepsilon} E\left(g\left(\tilde{X}_{\tau, \alpha} \mid \mathcal{M}_{t}\right)(\omega) .\right.\right.
$$

Thus by the definition (4.1) and the relation $t \leq r<t+\varepsilon$ we get

$$
\begin{aligned}
& U_{t}^{\alpha}(\omega)=\underset{\tau: \tau \geq t}{\operatorname{ess} \sup } E\left(g\left(\tilde{X}_{\tau, \alpha} \mid \mathcal{M}_{t}\right)(\omega)\right. \\
& =\max \left\{\operatorname { e s s } _ { \tau : \tau \geq r } E \left(g\left(\tilde{X}_{\tau, \alpha} \mid \mathcal{M}_{t}\right)(\omega), \operatorname{ess~sup}_{\tau: t \leq \tau<t+\varepsilon} E\left(g\left(\tilde{X}_{\tau, \alpha} \mid \mathcal{M}_{t}\right)(\omega)\right\}\right.\right. \\
& =\underset{\tau: \tau \geq r}{\operatorname{ess} \sup } E\left(g\left(\tilde{X}_{\tau, \alpha} \mid \mathcal{M}_{t}\right)(\omega)\right. \text {. }
\end{aligned}
$$

Therefore we can replace the inequality in (5.1) with the quality: Namely for all $r \in$ $[t, t+\varepsilon)$, it holds that

$$
U_{t}^{\alpha}(\omega)=E\left(U_{r}^{\alpha} \mid \mathcal{M}_{t}\right)(\omega)
$$

Therefore the proof of this theorem is completed.

In Theorem 5.1, (i) means $g\left(\tilde{X}_{t, \alpha}\right)$ is the lower bound of the optimal rewards $U_{t}^{\alpha}$. The properties (ii) and (iii) are called 'supermartingale' and 'martingale' respectively in theory of stochastic processes(see [9]), and (ii) means the optimal rewards $\left\{U_{t}^{\alpha}\right\}_{t \geq 0}$ has the supermartingale property over all the time space $[0, \infty)$. Moreover, (iii) means the optimal rewards preserve the martingale property until the optimal stopping time $\sigma_{\alpha}^{*}$ defined by (4.2). In the rest of this section we discuss the optimality equations for the optimal reward process $\left\{U_{t}^{\alpha}\right\}_{t \geq 0}$. Let $L^{2}([0, \infty))$ be the space of continuous functions $u$. : $[0, \infty) \mapsto \mathbb{R}$ satisfying $\int_{0}^{\infty}\left(u_{r}\right)^{2} \mathrm{~d} r<\infty$ and $\lim _{t \rightarrow \infty} u_{t}=0$. Let $\mathcal{L}$ be the space of functions by

$$
\mathcal{L}:=\left\{u . \in L^{2}([0, \infty)) \mid u \text {. is differentiable on }[0, \infty) \text { and } \mathrm{d} u_{t} / \mathrm{d} t \in L^{2}([0, \infty))\right\}
$$

Then we write $A u_{t}:=-\mathrm{d} u_{t} / \mathrm{d} t$. For $t \geq 0$, we put a bilinear form on $\mathcal{L} \times \mathcal{L}$ by

$$
\langle u ., v \cdot\rangle_{t}=\int_{t}^{\infty} u_{r} v_{r} \mathrm{~d} r \quad \text { for } u ., v . \in \mathcal{L}
$$

Then the following Lemma 5.1 is trivial and we can easily check Lemma 5.2 using the integration by parts.

Lemma 5.1. For $u ., v ., w . \in \mathcal{L}, \mu \in \mathbb{R}$ and $t \geq 0$, the following (i) - (iii) hold.

(i) $\langle u ., v .\rangle_{t}=\langle v ., u .\rangle_{t}$.

(ii) $\langle u ., v .+w .\rangle_{t}=\langle u ., v \cdot\rangle_{t}+\langle u ., w .\rangle_{t}$.

(iii) $\langle u ., \mu v .\rangle_{t}=\mu\langle u ., v .\rangle_{t}$. 
Lemma 5.2. For $w . \in \mathcal{L}$ and $t \geq 0$, it holds that $\langle A w ., w \cdot\rangle_{t}=\frac{1}{2}\left(w_{t}\right)^{2} \geq 0$.

For a stochastic process $\left\{Y_{t}\right\}_{t \geq 0}$, we define the differential $A Y_{t}$ by a stochastic process:

$$
A Y_{t}(\omega):=\lim _{s \downarrow 0} \frac{Y_{t}(\omega)-Y_{t+s}(\omega)}{s}
$$

if the limit exists. The following theorem gives an optimality equation of the optimal fuzzy reward process $\left\{U_{t}^{\alpha}\right\}_{t \geq 0}$ by Dirichlet form ([1]).

Assumption B. It holds that $U^{\alpha}(\omega) \in \mathcal{L}$ and $g\left(\tilde{X}_{\cdot, \alpha}(\omega)\right) \in \mathcal{L}$ for almost all $\omega \in \Omega$ and all $\alpha \in(0,1]$.

Theorem 5.2 (Optimality equation). Suppose Assumption B hold. Let $\alpha \in(0,1]$. The optimal reward process $\left\{U_{t}^{\alpha}\right\}_{t \geq 0}$ is a unique solution satisfying the following three inequalities (5.3) - (5.5): For almost all $\omega \in \Omega$ and all $t \geq 0$,

$$
\begin{aligned}
& U_{t}^{\alpha}(\omega) \geq g\left(\tilde{X}_{t, \alpha}(\omega)\right) ; \\
& A U_{t}^{\alpha}(\omega) \geq 0: \\
& \left\langle A U^{\alpha}(\omega), U_{\cdot}^{\alpha}(\omega)-g\left(\tilde{X}_{\cdot, \alpha}(\omega)\right)\right\rangle_{t}=0 .
\end{aligned}
$$

Proof. (5.3) is trivial from Theorem 5.1(i). Let $\alpha \in(0,1]$. For almost all $\omega \in \Omega$, from Theorem 5.1(ii) and the bounded convergence theorem we have

$$
\begin{aligned}
E\left(A U_{t}^{\alpha} \mid \mathcal{M}_{t}\right)(\omega) & =E\left(\lim _{s \downarrow 0} \frac{\left.U_{t}^{\alpha}(\omega)-U_{t+s}^{\alpha} \mid \mathcal{M}_{t}\right)(\omega)}{s} \geq\right. \\
& =\lim _{s \downarrow 0} \frac{U_{t}^{\alpha}-E\left(U_{t+s}^{\alpha} \mid \mathcal{M}_{t}\right)(\omega)}{s} \geq 0 .
\end{aligned}
$$

Thus, since $A U_{t}^{\alpha}(\cdot)$ is $\bigcap_{r: r>t} \mathcal{M}_{r}$-measurable and $\mathcal{M}_{t}=\bigcap_{r: r>t} \mathcal{M}_{r}$ by the right-continuity of $\left\{\mathcal{M}_{t}\right\}_{t \geq 0}$, we obtain $A U_{t}^{\alpha}(\omega) \geq 0$ for all $\alpha \in(0,1]$. Therefore (5.4) holds. Further, if $U_{t}^{\alpha}(\omega)>g\left(\tilde{X}_{t, \alpha}(\omega)\right)$ for some $t$, then from Theorem 5.1(iii) we have $A U_{t}^{\alpha}(\omega)=0$ in a similar proof to (5.4). This implies (5.5) together with (5.3) and (5.4). Therefore $U_{t}^{\alpha}(\omega)$ satisfies $(5.3)-(5.5)$. Finally we prove uniqueness of the solutions of $(5.3)-(5.5)$. Let $u^{*}$. and $v^{*}$ be solutions of $(5.3)-(5.5)$. Then, since $v^{*} \geq g\left(\tilde{X}_{\cdot, \alpha}(\omega)\right)$ and $A u^{*} \geq 0$, we have

$$
\left\langle A u_{.}^{*}, v^{*}-g\left(\tilde{X}_{\cdot, \alpha}(\omega)\right)\right\rangle_{t} \geq 0 \text { for all } t \geq 0 .
$$

Therefore, since $\left\langle A u_{\cdot}^{*}, u_{\cdot}^{*}-g\left(\tilde{X}_{\cdot, \alpha}(\omega)\right)\right\rangle_{t}=0$ by Lemma 5.1, we get

$$
\left\langle A u_{\cdot}^{*}, u_{\cdot}^{*}-v_{\cdot}^{*}\right\rangle_{t}=\left\langle A u_{\cdot}^{*}, u_{\cdot}^{*}\right\rangle_{t}-\left\langle A u_{\cdot}^{*}, v_{\cdot}^{*}\right\rangle_{t} \leq\left\langle A u_{\cdot}^{*}, g\left(\tilde{X}_{\cdot, \alpha}(\omega)\right)\right\rangle_{t}-\left\langle A u_{\cdot}^{*}, g\left(\tilde{X}_{\cdot, \alpha}(\omega)\right)\right\rangle_{t}=0 .
$$

In the same way, we also obtain

$$
\left\langle A v_{.}^{*}, v^{*}-u_{.}^{*}\right\rangle_{t} \leq 0
$$


By Lemma 5.1, these two inequalities imply

$$
\left\langle A\left(u_{.}^{*}-v_{.}^{*}\right), u_{.}^{*}-v_{.^{*}}^{*}\right\rangle_{t}=\left\langle A u_{.}^{*}-A v_{.}^{*}, u_{.}^{*}-v_{.}^{*}\right\rangle_{t}=\left\langle A u_{.}^{*}, u_{.}^{*}-v_{.}^{*}\right\rangle_{t}+\left\langle A v_{.}^{*}, v^{*}-u_{.}^{*}\right\rangle_{t} \leq 0
$$

for all $t \geq 0$. Together with Lemma 5.2, we get $\frac{1}{2}\left(u_{t}^{*}-v_{t}^{*}\right)^{2}=0$ for all $t \geq 0$. Thus $u^{*}=v_{.}^{*}$. Therefore, (5.3) $-(5.5)$ has a unique solution $U^{\alpha}(\omega)$.

\section{An example in Markov case}

In order to illustrate the results of the optimal stopping models in previous sections, we consider an example where an owner finds an optimal timing to sell his own asset. Let $\left\{B_{t}\right\}_{t \geq 0}$ be a one-dimensional standard Brownian motion on $(\Omega, \mathcal{F}, P)$, and put a stochastic process $\left\{W_{t}\right\}_{t \geq 0}$ as follows: $W_{0}$ is a positive constant and

$$
W_{t}:=W_{0}+B_{t}, \quad t \geq 0
$$

Let a stochastic process $\left\{a_{t}\right\}_{t \geq 0}$ by $a_{t}:=\gamma W_{t}$ for $t \geq 0$, where $\gamma$ is a constant satisfying $0<\gamma<1$. Hence we give a fuzzy stochastic system by the following fuzzy random variables $\left\{\tilde{W}_{t}\right\}_{t \geq 0}$ :

$$
\tilde{W}_{t}(\omega)(x):=L\left(\left(x-W_{t}(\omega)\right) / a_{t}(\omega)\right)
$$

for $t \geq 0, \omega \in \Omega$ and $x \in \mathbb{R}$, where the shape function is triangular type $L(x):=$ $\max \{1-|x|, 0\}$ for $x \in \mathbb{R}$. Then their $\alpha$-cuts are

$$
\tilde{W}_{t, \alpha}(\omega)=\left[\tilde{W}_{t, \alpha}^{-}(\omega), \tilde{W}_{t, \alpha}^{+}(\omega)\right]=\left[W_{t}(\omega)-(1-\alpha) a_{t}(\omega), W_{t}(\omega)+(1-\alpha) a_{t}(\omega)\right] .
$$

The random variable $\left\{W_{t}\right\}_{t \geq 0}$ means 'the price process of his asset' in a market and the fuzzy random variable $\left\{\tilde{W}_{t}\right\}_{t \geq 0}$ means 'fuzzy values of the prices' when he sell it through some communication tools like Internet. Let a 'discount factor' $r(r>0)$ and let a 'maintenance cost' $c(c>0)$. We consider a fuzzy stopping problem in a fuzzy stochastic system $\left\{\tilde{X}_{t}\right\}_{t \geq 0}$ defined by

$$
\tilde{X}_{t}(\omega):=e^{-r t} \tilde{W}_{t}(\omega)-c t \quad \text { for } t \geq 0, \omega \in \Omega,
$$

The $\alpha$-cuts of $(6.4)$ are $\tilde{X}_{t, \alpha}(\omega)=\left[e^{-r t} \tilde{W}_{t, \alpha}^{-}(\omega)-c t, e^{-r t} \tilde{W}_{t, \alpha}^{+}(\omega)-c t\right]$. Let a linear ranking function $g([a, b]):=(2 a+b) / 3$ for $a, b \in \mathbb{R}$ satisfying $a \leq b$, where the owner's pessimisticoptimistic index is taken as $\lambda=2 / 3([10]) . g$ satisfies the properties (3.1) and (3.2), and we can easily check

$$
g\left(\tilde{X}_{t, \alpha}(\omega)\right)=e^{-r t}\left(W_{t}(\omega)-(1-\alpha) a_{t}(\omega) / 3\right)-c t, \quad \omega \in \Omega
$$

for $\alpha \in[0,1]$. Then $e^{-r t}$ means a 'discount rate' in the market. Let a stopping time $\xi_{\alpha}(\omega):=\inf \left\{t \geq 0 \mid g\left(\tilde{X}_{t, \alpha}(\omega)\right) \leq 0\right\}$ for $\omega \in \Omega$, which means 'the time of bankruptcy regarding the asset'. Taking into account the bankruptcy in this example, we put (4.1) as

$$
U_{t}^{\alpha}=\underset{\tau: \text { stopping times, } \tau \geq t}{\operatorname{ess} \sup _{\text {min }}} E\left(g\left(\tilde{X}_{\left.\min , \xi_{\alpha}\right\}, \alpha}\right) \mid \mathcal{M}_{t}\right) \quad \text { for } t \geq 0, \alpha \in[0,1] .
$$


Hence a stopping time $\tau$ means 'a time to sell the asset', and he wants to find the optimal timing to sell his asset before bankruptcy. From (6.6), the $\alpha$-optimal stopping time (4.2) with bankruptcy becomes

$$
\begin{aligned}
\sigma_{\alpha}^{*}(\omega) & =\inf \left\{t \geq 0 \mid U_{\min \left\{t, \xi_{\alpha}\right\}}^{\alpha}(\omega)=g\left(\tilde{X}_{\min \left\{t, \xi_{\alpha}\right\}, \alpha}(\omega)\right)\right\} \\
& =\min \left\{\inf \left\{t \geq 0 \mid U_{t}^{\alpha}(\omega)=g\left(\tilde{X}_{t, \alpha}(\omega)\right)\right\}, \xi_{\alpha}(\omega)\right\}
\end{aligned}
$$

Next we check Assumption A. Let $\alpha, \alpha^{\prime} \in[0,1]$ satisfy $\alpha^{\prime} \leq \alpha$ and let $\omega \in \Omega$. Suppose $g\left(\tilde{X}_{t, \alpha^{\prime}}(\omega)\right)=U_{t}^{\alpha^{\prime}}(\omega)$ for some $t<\xi_{\alpha}(\omega)$. Since $\left\{e^{-r \min \left\{t, \xi_{\alpha}\right\}} W_{\min \left\{t, \xi_{\alpha}\right\}}\right\}_{t \geq 0}$ is a nonnegative supermartingale, by the optional sampling theorem $([3])$ we have

$$
\begin{aligned}
g\left(\tilde{X}_{t, \alpha}(\omega)\right) & =e^{-r t}\left(W_{t}(\omega)-(1-\alpha) a_{t}(\omega) / 3\right)-c t \\
& =e^{-r t}\left(W_{t}(\omega)-\left(1-\alpha^{\prime}\right) a_{t}(\omega) / 3\right)-c t-e^{-r t}\left(\alpha^{\prime}-\alpha\right) a_{t}(\omega) / 3 \\
& =g\left(\tilde{X}_{t, \alpha^{\prime}}(\omega)\right)-e^{-r t}\left(\alpha^{\prime}-\alpha\right) a_{t}(\omega) / 3 \\
& =U_{t}^{\alpha^{\prime}}(\omega)-e^{-r t}\left(\alpha^{\prime}-\alpha\right) \gamma W_{t}(\omega) / 3 \\
& \geq E\left(g\left(\tilde{X}_{\min \left\{\tau, \xi_{\alpha}\right\}, \alpha^{\prime}}\right) \mid \mathcal{M}_{t}\right)(\omega)-E\left(e^{-r \min \left\{\tau, \xi_{\alpha}\right\}}\left(\alpha^{\prime}-\alpha\right) \gamma W_{\min \left\{\tau, \xi_{\alpha}\right\}} / 3 \mid \mathcal{M}_{t}\right)(\omega) \\
& =E\left(g\left(\tilde{X}_{\min \left\{\tau, \xi_{\alpha}\right\}, \alpha^{\prime}}\right)-e^{-r \min \left\{\tau, \xi_{\alpha}\right\}}\left(\alpha^{\prime}-\alpha\right) a_{\min \left\{\tau, \xi_{\alpha}\right\}} / 3 \mid \mathcal{M}_{t}\right)(\omega) \\
& =E\left(g\left(\tilde{X}_{\min \left\{\tau, \xi_{\alpha}\right\}, \alpha}\right) \mid \mathcal{M}_{t}\right)(\omega), \quad \text { almost all } \omega \in \Omega
\end{aligned}
$$

for all bounded stopping times $\tau$ such that $\tau \geq t$. It follows $g\left(\tilde{X}_{t, \alpha}(\omega)\right)=U_{t}^{\alpha}(\omega)$. Therefore by (4.2) we obtain $\sigma_{\alpha}^{*}(\omega) \leq \sigma_{\alpha^{\prime}}^{*}(\omega)$ for almost all $\omega \in \Omega$, and Assumption A is fulfilled. We also have $E\left(\sup _{0 \leq t<\infty} \tilde{X}_{t, 0}^{+}\right) \leq E\left(\sup _{0 \leq t<\infty}\left(2 e^{-r t}\left(W_{0}+B_{t}\right)-c t\right)\right)<\infty$ from [3, Chap.3]. Setting $\left.f^{\alpha}(x):=x-(1-\alpha) \gamma x / 3(x \geq 0)\right)$, we obtain the optimal value function

$$
\begin{aligned}
V^{\alpha}(y) & =\sup _{\tau \geq 0} E\left(g\left(\tilde{X}_{\min \left\{\tau, \xi_{\alpha}\right\}, \alpha}\right) \mid W_{0}=y\right) \\
& =\sup _{\tau \geq 0} E\left(e^{-r \min \left\{\tau, \xi_{\alpha}\right\}} f^{\alpha}\left(W_{\min \left\{\tau, \xi_{\alpha}\right\}}\right)-c \min \left\{\tau, \xi_{\alpha}\right\} \mid W_{0}=y\right)
\end{aligned}
$$

for an initial price $y(y>0)$ of the asset. This function satisfies the following optimality equation (6.7) - (6.9) in Markov case ([9]):

$$
\begin{gathered}
V^{\alpha} \geq f^{\alpha} \\
-\frac{1}{2} \frac{\mathrm{d}^{2}}{\mathrm{~d} y^{2}} V^{\alpha}+r V^{\alpha} \leq c ; \\
-\frac{1}{2} \frac{\mathrm{d}^{2}}{\mathrm{~d} y^{2}} V^{\alpha}+r V^{\alpha}=c \quad \text { outside } B^{\alpha},
\end{gathered}
$$

where $B^{\alpha}:=\left\{y \in(0, \infty) \mid V^{\alpha}(y)=f^{\alpha}(y)\right\}$. Then, since the example is a Markov case, the $\alpha$-optimal stopping time $\sigma_{\alpha}^{*}(\omega)$ is reduced to

$$
\sigma_{\alpha}^{*}(\omega)=\min \left\{\inf \left\{t \geq 0 \mid W_{t}(\omega) \in B^{\alpha}\right\}, \xi_{\alpha}\right\}
$$


which is the first hitting time of the stopping region $B^{\alpha}$ by the stochastic process $\left\{W_{t}\right\}_{t \geq 0}$. The conditions (6.7)-(6.9) are corresponding to (5.3) - (5.5) in Theorem 5.2. Hence, (6.7) means $f^{\alpha}$ is the lower bound of the optimal value function $V^{\alpha}$. The properties (6.8) and (6.9) are called 'superharmonic' and 'harmonic' respectively in theory of Markov processes(see [9]). (6.8) means the optimal value function $V^{\alpha}$ is superharmonic over all the state space $(0, \infty)$, and (6.9) means the optimal value function is harmonic outside the stopping region $B^{\alpha}$. Clearly we have $\sigma_{\alpha}^{*}<\infty$ since $c>0$. Therefore the optimal fuzzy stopping time in Problem 1 is

$$
\begin{aligned}
\tilde{\sigma}^{*}(t, \omega) & =\sup _{\alpha \in[0,1]} \min \left\{\alpha, 1_{\left\{\sigma_{\alpha}^{*}>t\right\}}(\omega)\right\} \\
& =\sup \left\{\alpha \in[0,1] \mid V^{\alpha}\left(W_{t}(\omega)\right)>f^{\alpha}\left(W_{t}(\omega)\right) \text { and } t<\xi_{\alpha}(\omega)\right\}
\end{aligned}
$$

for $t \geq 0$ and $\omega \in \Omega$, where $f^{\alpha}(x)=x-(1-\alpha) \gamma x / 3(x \geq 0)$ and and the supremum of the empty set is understood to be 0 . This is the optimal timing to sell the asset.

\section{Concluding remarks}

In this paper, we have considered the stopping problem by means of fuzzy stopping times in a continuous-time fuzzy stochastic system, and the optimization is discussed through the scalarization method with linear ranking functions. The optimal fuzzy stopping time is constructed from a family of non-fuzzy stopping times which are characterized by the optimality equation at each grade $\alpha \in[0,1]$.

The fuzzy stopping time is one of natural extensions of the classical stopping ones by fuzzification. Since the fuzzy stopping time is a kind of vague decision by linguistically qualified statement, we need to demonstrate the actual algorithm/procedure of stopping rules in real applications. For the further works, it is interesting for us to investigate the above problem in case studies of particular application models, for example, group decision making, sequential stopping games, the option models in financial engineering and so on.

\section{Acknowledgments}

The authors would like to thank the referees for valuable comments and suggestions.

\section{References}

[1] A.Bensoussan and J.-L. Lions, Sur la théorie du contrôle optimal, 1: Temps d'arrêt (Hermann, Paris, 1977).

[2] A.G.Fakeev, Optimal stopping rules for processes with continuous parameter, Theory Probab. Appli. 15 (1970) 324-331.

[3] I.Karatzas and S.E.Shreve, Brownian motion and stochastic calculus (Springer, New York, 1991). 
[4] M.Kurano, M.Yasuda, J.Nakagami and Y.Yoshida, A limit theorem in some dynamic fuzzy systems, Fuzzy Sets and Systems 51 (1992) 83-88.

[5] M.Kurano, M.Yasuda, J.Nakagami and Y.Yoshida, An approach to stopping problems of a dynamic fuzzy system, Fuzzy Sets and Systems 131 (2002) 225-233.

[6] E.L.Presman and I.M.Sonin, The best choice problem for a random number of objects, Theor. Prob. Appl. 17 (1972) 657-668.

[7] M.L.Puri and D.A.Ralescu, Fuzzy random variables, J. Math. Anal. Appl. 114 (1986) 409-422.

[8] P.Fortemps and M.Roubens, Ranking and defuzzification methods based on area compensation, Fuzzy Sets and Systems 82 (1996) 319-330.

[9] A.N.Shiryayev, Optimal Stopping Rules (Springer, New York, 1979).

[10] X.Wang and E.E.Kerre, Reasonable properties for the ordering of fuzzy quantities (I), Fuzzy Sets and Systems 118 (2001) 375-385.

[11] G.Wang and Y.Zhang, The theory of fuzzy stochastic processes, Fuzzy Sets and Systems 51 (1992) 161-178.

[12] Y.Yoshida, The optimal stopped fuzzy rewards in some continuous-time dynamic fuzzy systems, Math. and Comp. Modelling 26 (1997) 53-66.

[13] Y.Yoshida, M.Yasuda, J.Nakagami and M.Kurano, Optimal stopping problems in a stochastic and fuzzy system, J. Math. Analy. and Appl. 246 (2000) 135-149.

[14] L.A.Zadeh, Fuzzy sets, Inform. and Control 8 (1965) 338-353. 